\title{
EL CONTROL INTERNO COMO INSTRUMENTO DE GESTIÓN DE LIQUIDEZ EMPRESARIAL: UN ANÁLISIS META-ANALÍTICO EN EL CANTÓN LATACUNGA
}

\author{
Ruiz Yadira ${ }^{1}$, Valenzuela Viviana ${ }^{2}$ y Tapia Julio $^{3}$. \\ $\left\{\right.$ ygruiz $^{1}$, vmvalenzuela $^{2}$, jctapia3 $\left.^{3}\right\} @$ espe.edu.ec \\ https://orcid.org/ 0000-0001-5254-13761' ${ }^{1}, 0000-0002-2707-7775^{2}, 0000-0001-9632-8550^{3}$. \\ Universidad de las Fuerzas Armadas ESPE \\ Sangolquí-Ecuador
}

Recibido (05/10/20), Aceptado (20/10/20)

\begin{abstract}
Resumen: En las organizaciones se presentan varias debilidades vulnerables en los sistemas de control interno y en la manera en que las empresas gestionan su liquidez, de modo, que el control interno debe garantizar la efectividad de los objetivos económicos empresariales. El objetivo de este trabajo es analizar el constructo teórico del control interno mediante el modelo COSO, a través de un análisis meta-analítico. Adicionalmente, se aplicólatécnicaestadística demeta-análisis, conelfin de evaluarelniveldesignificancia y su sesgo de error, por tal razón se direccionó bajo un diseño metodológico mixto. Los resultados revelaron que el factor ambiente de control, es descuidado por parte de las organizaciones debido al empirismo que maneja el directorio administrativo de las pequeñas y medianas empresas. Se concluye que el modelo $\mathrm{COSO}$, si mantiene relación consistente con el control interno y la gestión de liquidez empresarial.
\end{abstract}

Palabras Clave: Control interno, liquidez, gestión empresarial.

\section{INTERNAL CONTROL AS A CORPORATE LIQUIDITY MANAGEMENT INSTRUMENT: A META-ANALYTICAL ANALYSIS IN THE CANTON LATACUNGA}

\begin{abstract}
In organizations, there are several vulnerable weaknesses in internal control systems and in the way companies manage their liquidity, so that internal control must guarantee the effectiveness of business economic objectives. The objective of this work is to analyze the theoretical construct of internal control through the COSO model, through a meta-analytical analysis. Additionally, the statistical technique of meta-analysis was applied, in order to evaluate the level of significance and its error bias, for this reason it was conducted under a mixed methodological design. The results revealed that the control environment factor is neglected by organizations due to the empiricism managed by the administrative board of small and medium-sized companies. It is concluded that the COSO model, if it maintains a consistent relationship with internal control and business liquidity management.
\end{abstract}

Keywords: Internal control, liquidity, business management. 


\section{I.INTRODUCCIÓN}

En diferentes organizaciones se presentan varias debilidades vulnerables en los sistemas de control interno y en la forma en que las empresas gestionan la liquidez, sin embargo, el control interno debe adaptarse a las modificaciones que presentan las organizaciones empresariales con el propósito de garantizar la efectividad de los objetivos, verificar la confiabilidad de los informes y promover la adhesión de leyes y políticas [1]. No obstante, existen varias cuestiones de preocupación por parte de los directores financieros que buscan abordar temas como la liquidez de las empresas para mantener el nivel y valor empresarial [2]. Se considera que, en Ecuador la aplicación del control interno ha evolucionado dentro de los marcos legales para las empresas públicas y privadas [3]. Sin embargo, pueden presentar múltiples dificultades para frenar fraudes corporativos, lo cual, hace que esta aplicación del sistema tenga poca efectividad. Por otra parte el desarrollo económico del cantón Latacunga, se ha visto afectado por las malas prácticas de control interno, exponiendo que grandes riesgos generen bajos niveles de desempeño, disminuyendo la rentabilidad y ocasionando problemas de liquidez [4].

Ejecutar la idea de una propuesta de revisión sistemática es una excelente oportunidad para examinar prácticas pasadas y buscar eficiencias, mejoras a los problemas del control interno y gestión de liquidez empresarial [5]. Para lo cual, esta investigación requiere de importante información científica para contribuir con el conocimiento y desarrollo científico.

El control interno se originó por el inadecuado uso de recursos a nivel organizacional. De esta manera, los controles administrativos, financieros, han ido evolucionando en la escala organizacional como lo es el sistema de control interno COSO (Committee of Sponsoring Organizations of the Treadway) [6]. Éste comite fue creado con el propósito de definir al control interno como un proceso efectuado por el consejo de administración, la dirección y el resto del personal de una entidad, diseñado con el objetivo de proporcionar un grado de seguridad razonable [7]. Este sistema de control garantiza el cumplimiento de efectividad y eficiencia de las operaciones, confiabilidad de la información financiera y el cumplimiento de las leyes y regulaciones aplicables [8]. El marco COSO, visualiza cinco componentes principales del control interno. Cada uno de los componentes tiene una relación y puede influir en el funcionamiento de cualquier otro componente operado de una manera eficaz. El objetivo del presente artículo es analizar el constructo teórico del control interno mediante el modelo COSO, a través de un análisis me- ta-analítico.

El desarrollo de esta investigación, se encuentra estructurada de la siguiente manera: I. Introducción, se contextualizó el principal problema que enfrentan las organizaciones con relación al control interno y la gestión de liquidez empresarial. II. Desarrollo, a través de la revisión literaria permitió identificar el desarrollo, evolución del control interno en relación al modelo COSO y la gestión de liquidez empresarial. III. Metodología, en este apartado se describió el proceso de la estrategia de búsqueda de acuerdo a los criterios de inclusión y exclusión, se determinó los enfoques metodológicos y por último se identificó el total de la muestra analizada. IV. Resultados, se consideró las características principales de los estudios como bases científicas, orientación, publicación por años y mediante la técnica estadística, conocida como meta- análisis, se comprobó el nivel de significancia y sesgo de error del modelo. V. Conclusiones, se consideró evidencias, hallazgos generales con relación a la revisión sistemática y la probabilidad del éxito del modelo COSO.

\section{II.DESARROLLO}

El control interno está diseñado para que las empresas logren sus metas y objetivos en un determinado tiempo, a través de su funcionamiento busca promover la eficiencia y eficacia, enmarcado siempre en el cumplimiento de la normativa, además, apoya al crecimiento y desarrollo empresarial, el cual debe estar asociado al desempeño del personal en las operaciones, de esta manera se reducen los riesgos y se contribuye a la confiabilidad financiera y operacional [9].

El modelo COSO propone una estructura de control, que impulsa una nueva cultura administrativa y es la base para diversos modelos de control a nivel internacional [10], sin embargo, el control interno de manera general sirve para programar y ejecutar la autoevaluación de su actividad y operación. El modelo COSO es utilizado por las empresas, y en la presente investigación va ser motivo de análisis en relación al sistema de control interno, como herramienta para una adecuada gestión de liquidez. Según [11] menciona que el modelo COSO está compuesto por cinco componentes interrelacionados. (Ver figura 1). 


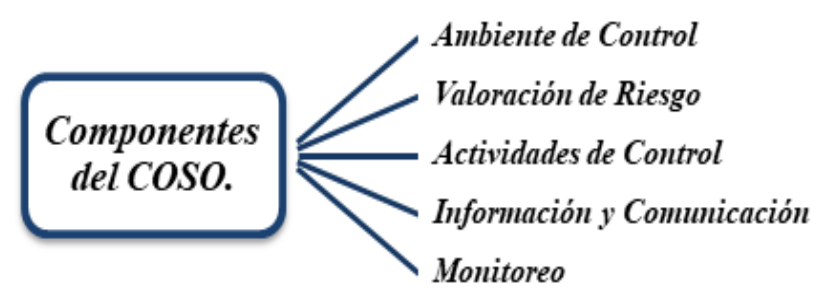

Fig. 1. Componentes del modelo COSO.

\section{A.Ambiente de Control.}

Es considerado la base fundamental del resto de componentes ya que permite determinar la estrategia, objetivos, actividades de una empresa e identificar, valorar y actuar sobre los riesgos. Sus factores son: integridad y valores éticos, orientación moral, compromiso para la competencia, comité de auditoría, estructura de la organización, filosofía y estilo de operación de la gerencia, políticas y prácticas de recursos humanos, asignación de autoridad y responsabilidad [11].

\section{B.Valoración de Riesgo.}

El proceso de valoración de los riesgos de una entidad, debe identificar y analizar las implicaciones de los riesgos relevantes, tanto para la entidad como para cada una de las actividades, permite valorar y considerar los eventos potenciales que pueden afectar el logro de los objetivos [11]. Se debe tener en cuenta los riesgos próximos y remotos que influye en la consecución de los objetivos, no obstante, se identifica y evalúa las posibles respuestas al riesgo transferir, compartir, reducir y aceptar. Riesgos próximos: (tengo control sobre el riesgo) riesgo laboral, riesgo financiero, riesgo de negocio, riesgo operacional, riesgo legal. Riesgo remoto: fenómenos naturales, política gubernamental, competencia [12].

\section{C.Actividades de Control.}

Las actividades de control, ayudan a asegurar que se llevan a cabo aquellas acciones identificadas como necesarias para afrontar los riesgos y así conseguir alcanzar los objetivos de la empresa. Dentro de sus principales elementos podemos encontrar, aprobaciones, autorizaciones, verificaciones (inventarios), conciliaciones, revisiones (registros adecuados de transacciones), seguridad (contratación de seguros), etc. [11].

\section{D.Información y Comunicación.}

Se identifica, recoge, procesa y presenta por medio de los sistemas de información. La comunicación es inherente al proceso de información. A través de una comunicación efectiva en todos los niveles, se puede lograr que la información se pueda valorar para poder combatir al riesgo y operar y lograr los objetivos. Sus principales características son: contenido apropiado, Información oportuna, Información actual, Información exacta, Información accesible [11].

\section{E.Monitoreo.}

Este componente valora la existencia, funcionamiento y la calidad del desempeño en el tiempo de los riesgos, comunicaciones, recomendaciones, capacitaciones con el fin de verificar si son confiables y generan resultados. Entre sus principales aspectos se encuentra el alcance y frecuencia, ¿quién evalúa?, procesos de evaluación, fuentes de información, ¿a quién se informa, metodologías? [11].

Así mismo, los procedimientos especifican y detallan un proceso, los cuales conforman un conjunto ordenado de operaciones o actividades determinadas secuencialmente en relación con los responsables de la ejecución, que deben cumplir políticas y normas establecidas señalando la duración y el flujo de documentos, por ejemplo: procedimiento para pago de nómina, cobro de cuentas, entre otras [13].

\section{F.Gestión Empresarial}

La Gestión Empresarial, se considera como la actividad ejercida por la persona al mando de la empresa que mejora la productividad y por ende la competitividad de las empresas o negocios. La gestión busca hacer las cosas mejor y rápido. En ese sentido, es necesario identificar los procesos que influyen en el éxito de la empresa, haciendo referencia a las medidas estratégicas, siendo factible para la organización proporcionando un adecuado manejo de sus recursos, buscando la creación de valor [14].

\section{G.Gestión de liquidez empresarial.}

La liquidez de las empresas es necesaria para un funcionamiento eficiente. Constituye uno de los criterios que permite reconocer la fortaleza financiera a clientes, proveedores, bancos y dueños. Actualmente, se identifican empresas que alcanzan determinados niveles de rentabilidad que las hacen prometedoramente competitivas [15]. La liquidez de una empresa se juzga por la agilidad que tiene para cumplir con sus obligaciones a corto y mediano plazo, es decir, la habilidad con la que cuenta la empresa para efectuar el pago de las obligaciones [16] [17].

\section{METODOLOGÍA}

La metodología del estudio inició con una revisión sistemática, la cual, se caracterizó por diseñar estrategias 
de búsqueda de acuerdo a las variables control interno y gestión de liquidez. Posteriormente, se seleccionaron artículos científicos de carácter empírico y sistemático, con el fin de analizar el modelo COSO con sus cinco componentes principales (ambiente de control, valoración al riesgo, actividades de control, información y comunicación y monitoreo) [5]. Adicionalmente, se aplicó el modelo estadístico del meta-análisis, con el propósito de integrar el constructo teórico del modelo con su sesgo de error [9]. Sin embargo, la objetividad y eficacia del meta- análisis dependió particularmente de la revisión sistemática [10].

El enfoque de investigación fue mixto, es decir, el método cualitativo estudió las principales fuentes teóricas, a través de la estrategia de búsqueda y selección de escritos publicados según criterios de inclusión como bases indexadas, idioma, orientación y se excluyeron estudios científicos no frecuentes (Ver Tabla 1). Por otra parte, el método cuantitativo, analizo los efectos de las investigaciones publicadas con relación a la calidad del estudio.

Los resultados estadísticos del meta- análisis fueron procesados por el software libre Forest Plot Generator, este programa elaboró Forest Plot (Parcelas Forestales), donde aprecia las tendencias de los efectos que existe entre los estudios, es decir que detectó la heterogeneidad, peso de los estudios y los intervalos de confianza [11]. Para realizar el análisis se incluyó una muestra total de 53 estudios seleccionados a través de la estrategia de búsqueda.

TABLA I. Estrategia de búsqueda (criterios de inclusión y exclusión).

\begin{tabular}{lcllll}
\hline \multicolumn{4}{c}{ ESTRATEGIA DE B ÚSQUEDA } & \\
\hline Criterios de inclusión & \multirow{2}{*}{ Criterios de exclusión } \\
\hline Bases indexadas & Idiomas & Orientación & & \\
Scopus & Español & Empírica & Tesis & \\
Web of science & Inglés & Revisión & Bases no indexadas & \\
Latindex & Portugués & sistemática & Sitios web & no \\
Libros & & & concurridos & \\
\hline
\end{tabular}

\section{IV.RESULTADOS}

La estructura de esta investigación, se da a partir de un análisis descriptivo enmarcado en la obtención de las principales características de la base de datos recopilada, para así conseguir la evolución de escritos conforme la orientación del estudio, frecuencia de publicación por año y bases indexadas.

De acuerdo al análisis de revisión de la literatura, se pudo determinar en la figura 2 que el $38 \%$ de los escritos revisados pertenecen a la base científica Scopus, por poseer investigaciones con mayor rigurosidad científica y metodológica con relación a las variables de estudio, es decir, esta base de datos cuenta con citas y resúmenes de alta calidad en la web apoyando a los campos de la ciencia y la tecnología. Mientras tanto, el 19\% corresponde a Web of Science, que cuenta con artículos de alto rendimiento científico, la cual, da eficacia y consistencia a los resultados, por último, el 6\% corresponde a libros, que apoyaron efectivamente a los análisis descriptivos realizados (Ver Figura 2).

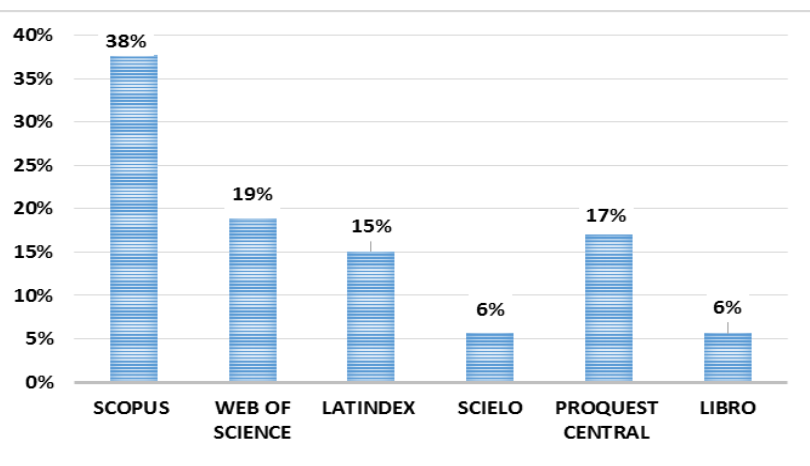

Fig. 2. Resultado de escrutinio de escritos científicos indexados.

Por otro lado, se identificó que el $96 \%$ de la muestra representa estudios de tipo empírico, es decir que la mayoría de las investigaciones son de campo con aportes significativos para validar el modelo, mientras que, el $4 \%$ de la búsqueda, cuenta con artículos orientados a la revisión sistemática.

De acuerdo a la frecuencia de publicación por año, la figura 3 determinó que el control interno y gestión de liquidez empresarial desde el año 2005, tienen un mí- 
nimo nivel de investigación. A partir de los años 2010 al 2019, se han publicado una gran variedad de escritos según las variables de estudio (Ver Figura 3). Esto indica que, durante este lapso de tiempo los estudios se enfocaban en analizar los fracasos y éxitos de los sistemas de control interno, evaluando las violaciones y abusos que surgían dentro de una organización. Es por ello, que estos escritos establecieron hallazgos para establecer respuestas y medidas correctivas [12].

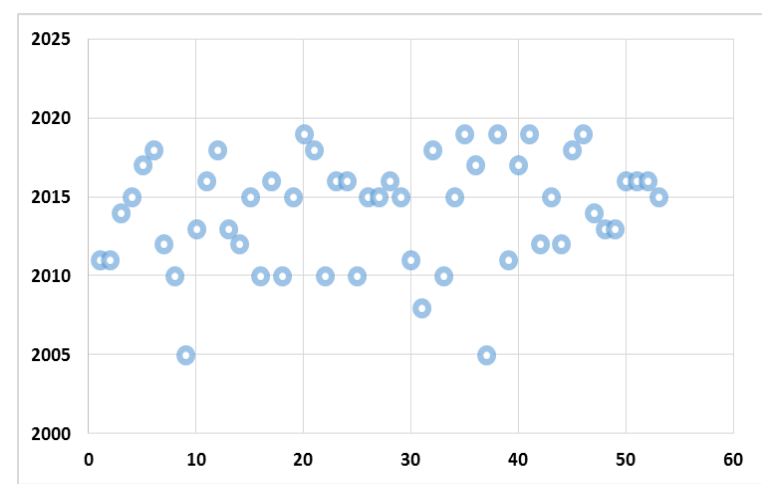

Fig. 3. Frecuencia de publicación por año

A fin de interpretar el análisis meta-analítico como técnica estadística, se elaboraron gráficos Florest Plot donde se detallan los resultados individuales por dimensión del modelo COSO, se consideraron los grados de heterogeneidad y homogeneidad, análisis de sensibilidad donde se observan los intervalos de confianza y error, para así comprobar si existe o no relación entre los estudios considerados dentro de la muestra.

En la figura 4 se expone a la dimensión I. Ambiente de control, se evidenció que la heterogeneidad es repetitiva, y su valor $\mathrm{p}$ estadístico es $\mathrm{p}=0,000$, esto significa que tiene alta relación con el modelo COSO. De acuerdo a la Figura 4, se observa que el compromiso por la competencia no se ha profundizado a través del modelo $\mathrm{COSO}$, por lo tanto los administradores que implementan los sistemas del control interno, no logran desarrollar un desempeño laboral competente y no se ajusta a los objetivos de cada organización [11]. Con respecto al análisis de artículos considerados, se evidencia que las medianas y pequeñas empresas del Ecuador, cantón Latacunga, han presentado problemas como inexpertica y falta de capacitación del personal, lo cual hace que esto tenga consecuencias en la gestión de liquidez [13].

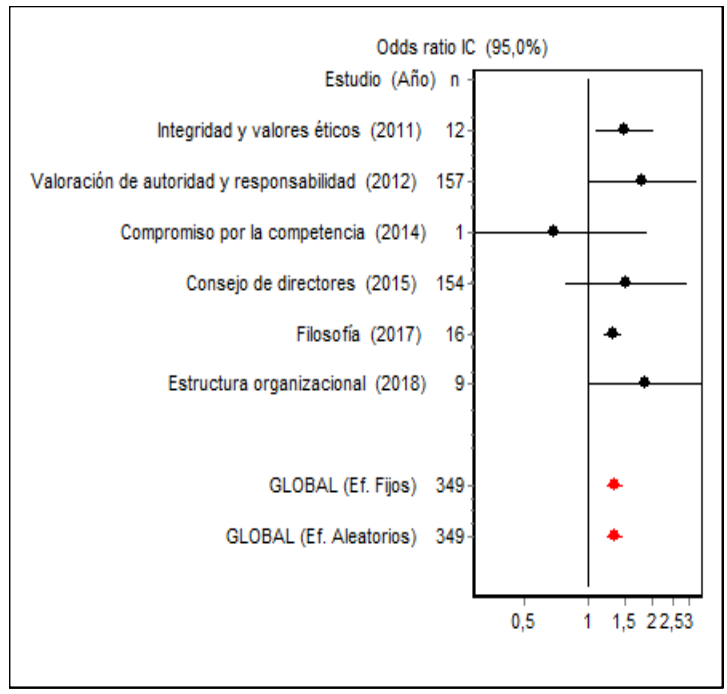

Fig. 4. Ambiente de Control (I) - Forest Plot.

Con relación a la dimensión II que consiste en la valoración del riesgo, se observó que la heterogeneidad persiste y su valor $\mathrm{p}=0,4800$ representa la relación entre la dimensión y el modelo COSO, sin embargo, presenta un cambio entre la sub dimensión identificación al riesgo (Ver Figura 5). Cabe determinar con base al análisis revisado, que las empresas no identifican el riesgo, porque no evalúan correctamente los procesos que influyen al cumplimiento de los objetivos, por lo tanto, sobreponen el nivel de riesgo en base a criterios personales, lo que hace que la identificación del riesgo no sea óptima. Las empresas del cantón Latacunga presentan problemas en el control de liquidez para el pago o cobro de sus obligaciones [13].

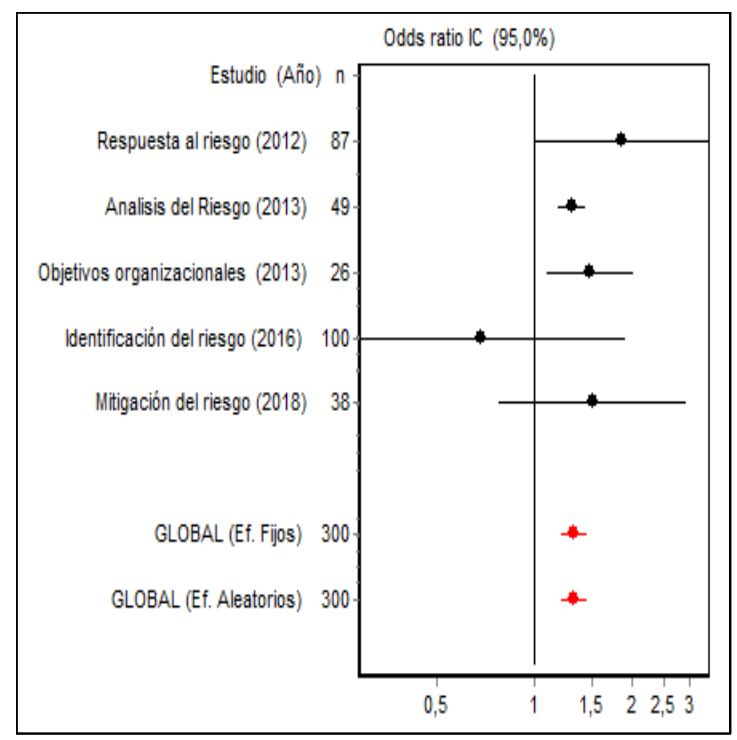

Fig. 5. Valoración al riesgo 8 (II) - Forest Plot. 
En lo que se refiere a la dimensión III Evaluada, referente a las actividades de control, se identificó que la homogeneidad es distante, no obstante, su valor $\mathrm{p}$ estadístico es igual a 0,3750 , lo que significa que la dimensión evaluada presentó leves inconsistencias teóricas (ver figura 6). En particular, esta dimensión identificó acciones con el único fin de afrontar riesgos y cumplir objetivos. Además, busca minimizar los efectos negativos como deficiencias en la liquidez [8]. De acuerdo al análisis efectuado, se pudo acotar que dentro de las empresas del cantón Latacunga, establecen políticas y reglamentos con la finalidad de controlar y dar cumplimiento a las funciones empresariales [14].

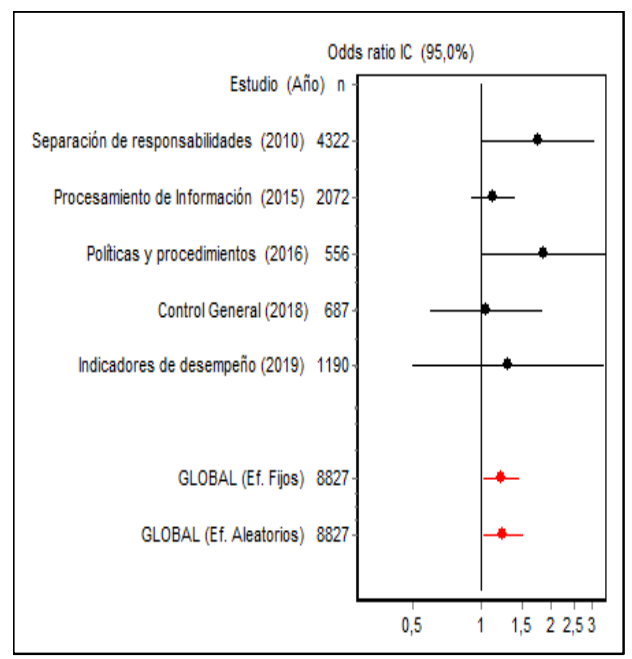

Fig. 6. Actividades de control (III) - Forest Plot.

La dimensión IV relacionada con la información y comunicación, reveló que existen factores homogéneos (Información administrativa, información operacional, política de comunicación, comunicación interna y comunicación externa.), con relación al análisis del constructo teórico. Sin embargo, se obtuvo un valor p estadístico de 0,486; que como efecto del factor información financiera, mantiene un sesgo de heterogeneidad, lo cual significa que la dimensión y el modelo COSO están relacionados, aunque presenta debilidad en el factor información financiera (ver figura 7). Se puede identificar que la comunicación e información financiera impartida, se enfoca a la confiabilidad de los resultados financieros que tiene la gerencia para una correcta toma de decisiones [15]. Según el análisis efectuado, se observó que en el cantón Latacunga las empresas consideran necesario que la información sea oportuna y acorde a los análisis financieros [16].

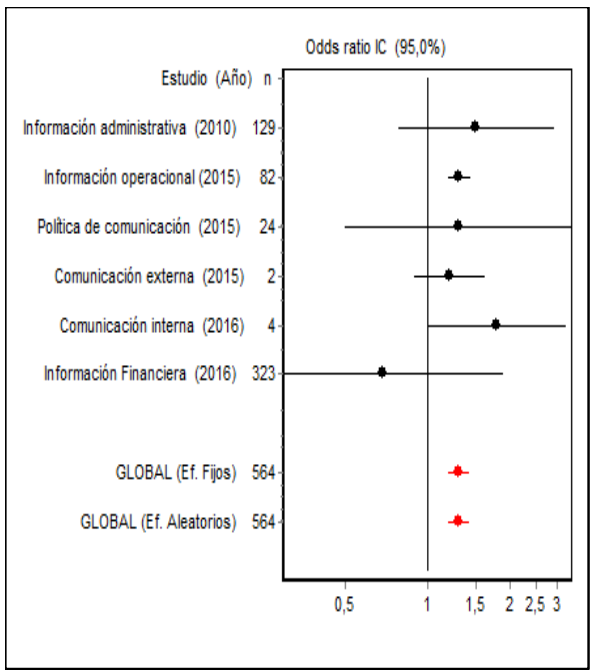

Fig. 7. Información y comunicación (IV) - Florest Plot

Dentro del análisis de la dimensión V referente al monitoreo, se evidenció la presencia de homogeneidad, a pesar de esto cuenta con un valor p estadístico de 0,9343; es decir que el componente monitoreo mantiene relación con el modelo, no obstante, no se desarrolla de la manera adecuada, por lo cual se evidencia un nivel significativo de error a pesar de mantener relación conceptual, no obstante, los lineamientos de control deben ser mejorados (ver figura 8). Las empresas que cuentan con un sistema de control interno, deben ejecutar procesos de monitoreo con el propósito de valorar la calidad y el desempeño de acuerdo a la consecución de objetivos y obligaciones [17]. De acuerdo al análisis realizado se destaca que las empresas del cantón Latacunga, consideran que es necesario tener supervisión en las distintas áreas, para así mantener un adecuado registro de actividades que de alguna manera aseguren el proceso del control interno [18]. 


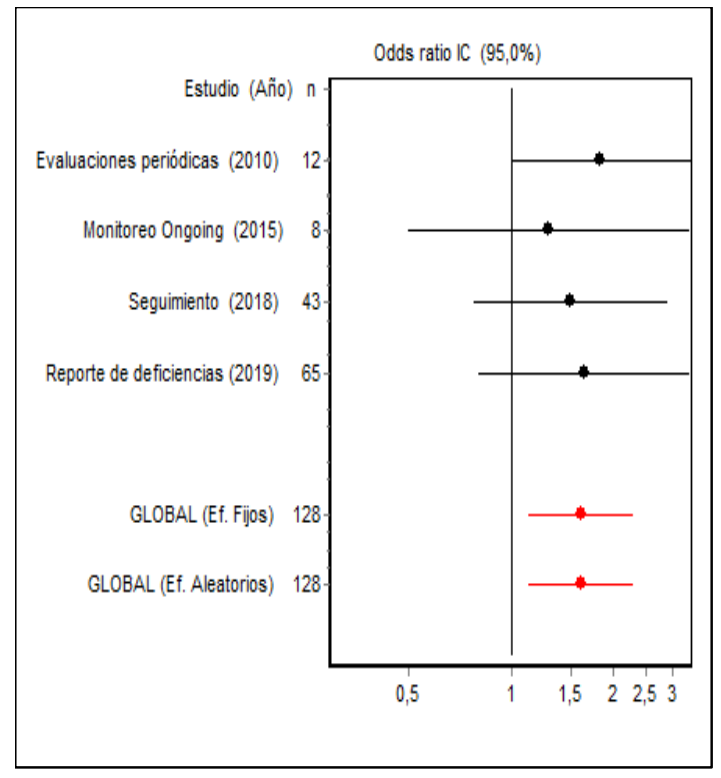

Fig. 8. Monitoreo (IV) - Florest Plot.

\section{V.CONCLUSIONES}

Se pudo concluir que del total de la muestra de cincuenta y tres estudios que se analizaron a través de una revisión sistemática, se identificó que la mayor parte de artículos seleccionados son empíricos y pertenecen a la base científica Scopus por poseer investigaciones con mayor rigurosidad científica y metodológica con relación a las variables de estudio el control interno y gestión de liquidez empresarial.

De acuerdo, a los resultados estadísticos arrojados por el meta-análisis se evidenció que existe leves inconsistencias en los factores evaluados dentro de cada componente, es decir que, el modelo COSO no enfatiza elementos como el compromiso por la competencia dentro del ambiente de control, identificación al riesgo en la valoración al riesgo e información financiera en la dimensión información y comunicación, sin embargo, esto no quiere decir que no sean analizados por ser poco relevantes si no por la falta de control en los procesos internos de las empresas.

En concordancia al modelo de estudio, se evidencia que la gestión de liquidez si guarda relación con el modelo COSO a partir del componente valoración al riesgo e información y comunicación por efectuar análisis de riesgos en los índices de liquidez y mantener un constante nivel de eficiencia de criterios que fortalecen la gestión empresarial. Por lo tanto, se concluye que el modelo COSO y sus cinco componentes si guarda una fuerte relación con el constructo teórico y las variables control interno y gestión de liquidez empresarial.

\section{REFERENCIAS}

[1]A. I. Calderón Peña y K. J. Jáuregui Salcedo, «El control interno y su relación con el cumplimiento de las obligaciones tributarias en las empresas comerciales, Chaclacayo, 2015,» Revista de Investigación Universitaria, vol. 5, $\mathrm{n}^{\mathrm{o}}$ 1, pp. 32-27, 2016.

[2]H. Almeida, M. Campello, I. Cunha y M. S. Weisbach, «Corporate Liquidity Management: A conceptual Framework and Survey» El Annual Review of Financial Economics, vol. 6, pp. 135-162, 2014.

[3]R. M. Cedeño Zambrano y L. M. Morell González, «La gestión de riesgos en Ecuador: una aproximación evolutiva desde el control interno,» Cofín Habana., vol. 12, no 2, pp. 306-318, 2018.

[4]M. P. Suntasig Tigse, El control interno del proceso de concesión de créditos y cobranzasy su incidencia en la liquidez de la empresa cereales La Pradera de la parroquia Belisario Quevedo, en el primer trimestre del año 2014, Latacunga: Universidad Técnica de Cotopaxi, 2014.

[5]L. Graham, Internal Control Audit and Compliance, Canadá: JohnWiley \& Sons, Inc., Hoboken, New Jersey. Published, 2015.

[6]S. Mantilla, Auditoría del control interno (3a. ed.), México: ECOE, 2013.

[7]N. Viloria, «Factores que inciden en el sistema de control interno de una organización.,» Actualidad Contable Faces, vol. 8, n 11, pp. 87-92, 2005.

[8]M. Samuel, Internal Control- Integrated Framework., México: ECOE, 2008.

[9]R. Estupiñán Gaitán, Administración de riesgos E.R.M. y la auditoría interna, 2006.

[10]G. Rivas Márquez, «Modelos contemporáneos de control interno. Fundamentos teóricos,» Observatorio Laboral Revista Venezolana, vol. 4, no 8, pp. 115-136, 2011.

[11]S. A. Mantilla B, Control Interno Informe COSO, 2017.

[12]L. R. Sánchez Sánchez, «COSO ERM y la Gestión de Riesgos.,» QUIPUKAMAYOC Revista de la Facultad de Ciencias Contables, vol. 23, $\mathrm{n}^{\circ}$ 44, pp. 43-50, 2015.

[13]M. E. Vivanco Vergara, «Los manuales de procedimientos como herramientas de control interno de una organización,» vol. 9, pp. 313-318, 2017.

[14]A. L. Quispe Otoma, M. P. Padilla Matínez y J. A. Telot, «Tecnologías de información y comunicación en la gestión empresarial de pymes comerciales,» vol. XXXVIII, nº 1, pp. 81-92, 2017.

[15]I. Domínguez García, «Evolución de la teoría sobre el análisis y gestión de la liquidez empresarial,» vol. 9, $\mathrm{n}^{\mathrm{o}} 1$, pp. 66-80, 2015. 
[16]L. Gitman y C. Zutter, Prinpios de administración financiera, México, 2012.

[17]F. Madrigal, S. Ayala y L. Chávez, «Análisis e interpretación de estados financieros en condiciones de incertidumbre,» vol. 2, p. 380, 2015.

[18]J. Sánchez, «Cómo realizar una revisión sistemática y un meta-análisis,» Aula abierta, vol. 38, n 2, pp. 53-64, 2010.

[19]D. Ojeda y J. Wurth, «Qué es un Meta análisis ?,» Revista Chilena de Anestesía, no 43, pp. 343-350, 2014. [20]J. A. González, E. Cobo y M. Villaró, «Revisión sistemática y meta-análisis,» Bioestadistica para no estadísticos, pp. 1-42, 2014.

[21]E. Osadchy y E. M. Akhmetshin, «Development of the financial control system in the company in crisis,» Mediterranean Journal of Social Sciences, vol. 6, $\mathrm{n}^{\circ}$ 552, pp. 390-398, 2015.

[22]I. C. Asubadin Azubadin, Control interno del Proceso de compras y su incidencia en la liquidez de la empresa comercial Yucailla Cia. Ltda. Latacunga durante el año 2010", Latacunga: Universidad Técnica de Ambato, 2011.

[23]J. C. Viera Quishpe, Diseño de un sistema de control interno administrativo y contable para el laboratorio autoservicio diésel de la sierra, ubicado en la ciudad de Latacunga, Latacunga: Universidad Central del Ecuador, 2015.

[24]M. Nava Rosillón, «Análisis financiero: una herramienta clave para una gestión financiera eficiente.,» Revista Venezolana de Gerencia, vol. 14, no 48, pp. 606628, 2009.

[25]S. P. Guayaquil Villarroel, El control interno y la razonabilidad de la información financiera en el Centro Comercial Popular de la ciudad de Latacunga en los locales comerciales de calzado en el período Enero - Junio 2016, Latacunga: Universidad Técnica de Ambato, 2015.

[26]A. Masli, G. E. Peters, V. J. Richardson y J. Sanchez, «Examining the potential benefits of internal control monitoring technology, " Accounting Review, vol. 85, no 3, pp. 1001-1034, 2010.

[27]D. C. Chasi Toca, "Gestión de riesgos y su incidencia en el control interno en el departamento financiero de la florícola fegacón cía. Ltda. Parroquia Joseguango bajo, cantón Latacunga, provincia de Cotopaxi", Latacunga: Universidad Técnica De Cotopaxi Facultad, 2017.

\section{RESUMEN CURRICULAR}

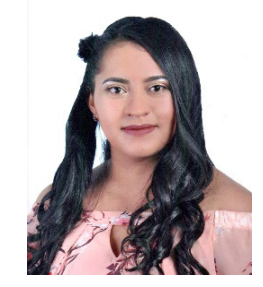

Yadira Ruiz, sus estudios universitarios los realizó en la Universidad de las Fuerzas Armadas ESPE Sede Latacunga, en el Departamento de Ciencias Económicas, Administrativas y del Comercio. Es candidata a Ingeniera en Finanzas, Contador Público y Auditor.

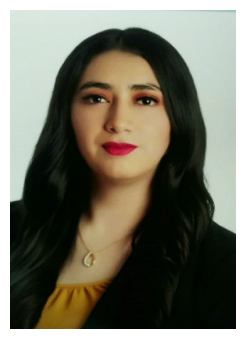

Viviana Valenzuela, sus estudios universitarios los realizó en la Universidad de las Fuerzas Armadas ESPE Sede Latacunga, en el Departamento de Ciencias Económicas, Administrativas y del Comercio. Es candidata a Ingeniera en Finanzas, Contador Público y Auditor.

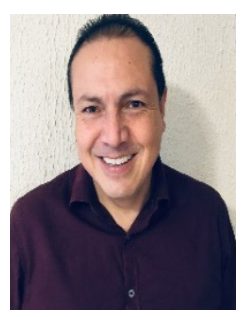

Julio Tapia, Magister en Administración de empresas, Magister ejecutivo en Dirección de Empresas, Ingeniero Comercial, Docente Titular de la Universidad de las Fuerzas Armadas ESPE Sede Latacunga 